\title{
Theory of wakefields in a dielectric-filled cavity
}

\author{
J. H. Kim, ${ }^{*}{ }^{\dagger}$ J. Han, ${ }^{\dagger}$ M. Yoon, and S. Y. Park ${ }^{\dagger}$ \\ Department of Physics, Pohang University of Science and Technology, Hyoja-dong, Nam-gu, Pohang, Gyeongbuk 790-784, Korea
} (Received 18 September 2009; published 15 July 2010)

\begin{abstract}
An analytical solution of a wakefield from a charge moving on the axis of a dielectric-filled cylindrical cavity is derived. A solution to the wakefield in a waveguide with only a boundary at the cavity entrance is already known. To take into account a boundary at the cavity exit, we introduce an imaginary antibeam, with opposite charge, which is created at the same time when the beam passes the exit boundary and continues to move along with the original beam at the same velocity. Although the beam has been annihilated in the net effect, the original beam and the antibeam produce their own wakefields, respectively, because they were created at different times. These superimposed fields are then mirror reflected as usual by the conducting exit boundary and the wakefield can be obtained by properly mirror reflecting them whenever it reaches a boundary. We find a resonance condition to enhance wakefields with multiple bunches of charges, and show that the acceleration gradient increases under that condition.
\end{abstract}

DOI: 10.1103/PhysRevSTAB.13.071302

PACS numbers: 41.60.- $\mathrm{m}$, 41.75.Ht, 41.75.Lx

\section{INTRODUCTION}

The problem of calculating wakefields in a waveguide loaded with dielectric layers has been solved for the case of an infinitely long waveguide and the possibility to apply it for a high gradient accelerator was discussed by several authors [1-5]. However, in reality, a waveguide is always bounded by both input and output ends forming a kind of a cavity. The boundary effects are important to calculate correct wakefields [6-9]. Furthermore, the cavity structure opens up a new interesting possibility to enhance further the accelerating gradient using a train of beam bunches. It has been demonstrated experimentally by Wang et al. that the maximum acceleration gradient was $1.3 \mathrm{GeV} / \mathrm{m}$ using a chemical vapor deposition diamond-lined test cavity [10].

Consider a cylindrical cavity bounded by conducting planes at $z=0$ and $z=d$, and assume that the cavity is filled with a dielectric. The problem can be solved in two steps: (1) by solving the semi-infinite waveguide problem bounded only by the input boundary, and (2) by including the effect of the output boundary.

The general problem to calculate the boundary effects can be traced back to Sommerfeld, Brillouin, and Rubinowicz [11-13]. The problem applied specifically to a semi-infinite dielectric-loaded waveguide has been discussed recently by Onishchenko et al. [6,7] taking into account an input end only. As far as the beam does not reach the end of the cavity, it never sees the exit boundary and thus the cavity wakefields are the same as those for a semi-infinite waveguide. In this paper, we also discussed the problem to calculate the entrance boundary effects. In

\footnotetext{
*kimrip@ postech.ac.kr

${ }^{\dagger}$ Permanent address: SP Microwave, Inc., 1107 JEI Platz, 45911 Gasan-dong, Geumcheon-gu, Seoul, Korea.
}

this case, our results are the same as those obtained by Onishchenko et al., who solved the problem in a rather complicated manner by performing a series of conformal mappings. In our paper, we obtained the same solution by performing just one simple conformal mapping. This mapping function used by us is similar to the one used by Rubinowicz [13] to solve a seismic wave problem. To the best of our knowledge, this is the first that uses this mapping function in a wakefield problem.

The purpose of the present paper is to extend the previous semi-infinite waveguide problem to a more realistic case, such that the wakefields due to a cylindrical cavity are derived. In order to solve this cavity wakefield problem, one must consider properly the exit boundary conditions in addition to the entrance boundary conditions. We accomplished this task by generalizing the image charge method. In the conventional image charge method used for an electrostatic problem, one can meet a conducting boundary condition in a rather straightforward manner by superimposing the mirror reflected fields from an image charge. However, in a wakefield problem, one needs a totally different image charge method. While the beam is still in the cavity, the beam does not see the exit boundary so that the solution is just the same as the one for a semi-infinite waveguide. After the beam exited the cavity, one can obtain the solution in the following way.

Let us suppose that an imaginary "antibeam" with opposite charge is created at the same time when the beam passes the exit boundary. Let us further suppose that this antibeam continues to move along with the "original beam" at the same velocity. Although, in net effect, the beam has been annihilated, the beam and the antibeam produce their own wakefields, respectively. Notice that the superimposed wakefields from these two beams are not trivial because they were created at different times. These superimposed fields are then mirror reflected as 
usual by the conducting exit boundary. This reflected field represents a wakefield propagating to the left with no source associated with it. The portion of the wakefield, which was generated from the original beam but still remained inside the cavity, continues to propagate to the right. As a result, one succeeded in obtaining nontrivial wakefield solutions that satisfy all the boundary conditions with no source associated inside the cavity.

If this left propagating wakefield reaches the entrance boundary, one can obtain the reflected wakefield just by performing a simple mirror reflection by the conducting entrance boundary, since no charge is associated with this field. The reflected wakefield now becomes a right moving wave. As time elapses, the wakefield continues bouncing back and forth between the two boundaries. In summary, the cavity wakefield can be obtained by properly mirror reflecting the superimposed wakefield from the original beam created at the entrance time and the antibeam created at the exit time.

In order to compare the results with the well-known vacuum solutions [14], we examined the low dielectricconstant limit of our solution; since our wakefield solution deals with the case that the phase velocity of the electromagnetic wave is less than the beam velocity, a dielectric material is necessary. When the beam just entered the cavity most flux lines emanating from the charge are concentrated in a thin quasispherical shell containing the beam. As the beam moves away from the entrance boundary, the flux lines stretch out to form a quasiplanar disk. These features agree with the well-known vacuum solution. Of course, since the wakefield condition is still met, some wakefield lines appear behind the Coulomb disk and remain even after the beam exited the cavity.

To apply the results for the wakefield accelerator, we try to enhance the acceleration gradient in a cavity using a train of beam bunches. In a waveguide or a semi-infinite waveguide, to enhance the wakefield using beam bunches, it is sufficient to consider only the time interval between each bunch, namely synchronization condition. In a cavity, however, the resonance condition such that Cerenkov radiation is superimposed constructively with itself, should be considered in addition. We demonstrate an acceleration gradient of $1.3 \mathrm{GV} / \mathrm{m}$ with ten bunches which have $2 \mathrm{nC}$ and $30 \mathrm{MeV}$ in a cavity filled with a dielectric of $\varepsilon=3$.

The organization of the paper is as follows. An analytical solution to the wakefield in a semi-infinite circular waveguide filled with a dielectric is derived in Sec. II. A complete solution to wakefields in a dielectric-filled cavity is given in Sec. III. The cavity problem is solved by applying the output boundary condition to the field solution of a semi-infinite waveguide. Here, we introduce an image method for a moving charge and a method of folding fields to make the solution satisfy the output boundary condition. A result and discussion on field enhancement for a high gradient accelerator is described in Sec. IV. Finally, a summary and discussion is given in Sec. V.

\section{WAKEFIELD IN A SEMI-INFINITE WAVEGUIDE}

In this section we revisit the problem of calculating the effect of the input boundary, a semi-infinite waveguide problem discussed by Onishchenko et al. [6,7]. Although the problem is physically the same, the mathematical approach introduced in this section for calculating the $I_{2}$ integral in Eq. (11) is considerably simpler than Ref. [6]. Then the following section deals with a more realistic cavity geometry so that the exit boundary is also treated.

Consider a dielectric-lined waveguide that is bounded at the input end only. The boundary is assumed to be a conducting plane which is thin enough to allow for the beam to pass through freely but which reflects the electromagnetic wave. Consider a ring-shaped beam injected, at $t=0$, into the waveguide bounded at $z=0$. The beam acts as a source term for the wave equations for $z>0$ and $t>0$, such that

$$
\rho=q_{b} \frac{\delta\left(r-r_{b}\right)}{2 \pi r} \delta\left(z-z_{b}\right) \quad J_{z}=\nu_{b} \rho, \quad \vec{J}_{\perp}=0,
$$

where the beam is assumed to be moving uniformly in time $t$, i.e., $z_{b} \equiv \nu_{b} t$.

The wave equations for $\mathrm{TM}_{\mathrm{on}}$ mode $\left(E_{z}, E_{r}, H_{\theta}\right)$ are given by

$$
\begin{aligned}
& {\left[\left(\frac{\partial^{2}}{\partial r^{2}}+\frac{1}{r} \frac{\partial}{\partial r}\right)+\frac{\partial^{2}}{\partial z^{2}}-\frac{\varepsilon \mu}{c^{2}} \frac{\partial^{2}}{\partial t^{2}}\right] E_{z}} \\
& \quad=\frac{4 \pi}{\varepsilon}\left(1-\varepsilon \mu \beta^{2}\right) \frac{\partial \rho}{\partial z} \\
& {\left[\left(\frac{\partial^{2}}{\partial r^{2}}+\frac{1}{r} \frac{\partial}{\partial r}-\frac{1}{r^{2}}\right)+\frac{\partial^{2}}{\partial z^{2}}-\frac{\varepsilon \mu}{c^{2}} \frac{\partial^{2}}{\partial t^{2}}\right]\left(\begin{array}{c}
E_{r} \\
H_{\theta}
\end{array}\right)} \\
& \quad=\frac{4 \pi}{\varepsilon}\left(\begin{array}{c}
1 \\
\varepsilon \beta
\end{array}\right) \frac{\partial \rho}{\partial r},
\end{aligned}
$$

where $\beta \equiv \nu_{b} / c$. One can expand the fields in terms of radial eigenmodes as

$$
\left(\begin{array}{c}
E_{z}(r, z, t) \\
E_{r}(r, z, t) \\
H_{\vartheta}(r, z, t)
\end{array}\right)=\frac{1}{2 \pi} \int d \omega e^{-i \omega t} \sum_{n=1}^{\infty}\left(\begin{array}{c}
a_{z n}(z) e_{z n}(r) \\
a_{r n}(z) e_{r n}(r) \\
a_{\theta n}(z) h_{\vartheta n}(r)
\end{array}\right),
$$

where $\left(e_{z n}, e_{r n}, h_{\theta n}\right)$ are the radial eigenmodes satisfying the source free wave equations,

$$
\begin{array}{r}
{\left[\left(\frac{d^{2}}{d r^{2}}+\frac{1}{r} \frac{d}{d r}\right)+k_{\perp n}^{2}\right] e_{z n}(r)=0} \\
{\left[\left(\frac{d^{2}}{d r^{2}}+\frac{1}{r} \frac{d}{d r}-\frac{1}{r^{2}}\right)+k_{\perp n}^{2}\right]\left(\begin{array}{c}
e_{r n}(r) \\
h_{\vartheta n}(r)
\end{array}\right)=0,}
\end{array}
$$

and the boundary condition $e_{z n}\left(R_{1}\right)=0$ on the waveguide wall. The solution of Eq. (4) can be found easily as 


$$
e_{z n}(r)=J_{0}\left(k_{\perp n} r\right),
$$

where $k_{\perp n} \equiv j_{0 n} / R_{1}$ and $j_{0 n}$ is the $n$th root of $J_{0}(x)=0$. Other radial eigenmodes are given in terms of Eq. (5a) as

$$
\left(\begin{array}{c}
e_{r n}(r) \\
h_{\vartheta n}(r)
\end{array}\right)=\frac{1}{k_{\perp n}^{2}}\left(\begin{array}{c}
k_{n} \\
\varepsilon \frac{\omega}{c}
\end{array}\right) e_{z n}^{\prime}(r) \quad\left(\prime \equiv \frac{d}{d r}\right),
$$

where the axial eigenvalues $k_{n}$ are given in terms of the radial eigenvalues $k_{\perp n}^{2}$ by the relation

$$
k_{n}^{2} \equiv \varepsilon \mu \frac{\omega^{2}}{c^{2}}-k_{\perp n}^{2} .
$$

Inserting Eq. (3) into Eq. (2) and using Eq. (4) along with the source given in Eq. (1), we have

$$
\begin{aligned}
& \sum_{n=1}^{\infty}\left(\frac{d^{2}}{d z^{2}}+k_{n}^{2}\right) a_{z n}(z) e_{z n}(r) \\
& =\frac{2 q_{b}}{\varepsilon}\left(1-\varepsilon \mu \beta^{2}\right) \frac{\delta\left(r-r_{b}\right)}{r} \frac{i \omega}{\nu_{b}^{2}} e^{i\left(\omega / \nu_{b}\right) z} \\
& \sum_{n=1}^{\infty}\left(\frac{d^{2}}{d z^{2}}+k_{n}^{2}\right)\left(\begin{array}{c}
a_{r n}(z) e_{r n}(r) \\
a_{\theta n}(z) h_{\theta n}(r)
\end{array}\right) \\
& =\frac{2 q_{b}}{\varepsilon}\left(\begin{array}{c}
1 \\
\varepsilon \beta
\end{array}\right)\left(\frac{\partial}{\partial r} \frac{\delta\left(r-r_{b}\right)}{r}\right) \frac{1}{\nu_{b}} e^{i\left(\omega / \nu_{b}\right) z}
\end{aligned}
$$

We previously proved a generalized orthogonality relation between two arbitrary modes on the dispersion lines [1]. Between the modes with the same $\omega$, the orthogonality relation can be written as

$$
\int_{0}^{R_{1}} \operatorname{drre}_{r n}(r) h_{\vartheta m}(r)=\delta_{n m} C_{n} \frac{\omega}{c} k_{n}
$$

where

$$
\begin{aligned}
C_{n} & =\frac{\varepsilon}{k_{\perp n}^{4}}\left[r e_{z n}^{\prime} e_{z n}+\frac{r^{2}}{2}\left(e_{z n}^{\prime 2}+k_{\perp n}^{2} e_{z n}^{2}\right)\right]_{r=R_{1}} \\
& =\frac{\varepsilon R_{1}^{2}}{2 k_{\perp n}^{2}} J_{1}^{2}\left(j_{0 n}\right) .
\end{aligned}
$$

Equation (6) can be solved in terms of a particular solution of the inhomogeneous equation with the source term and a general solution of the homogeneous equation without the source term. Defining the variable $k \equiv \frac{\omega}{\nu_{b}}$, the solution to Eq. (6) can be written as

$$
\begin{aligned}
\left(\begin{array}{c}
a_{r n}(z) \\
a_{\theta r n}(z)
\end{array}\right)= & 2 q_{b} \frac{e_{z n}\left(r_{b}\right)}{C_{n}} \frac{1}{\nu_{b}}\left(\begin{array}{c}
\frac{1}{k_{n}} \\
\frac{1}{k}
\end{array}\right) \frac{1}{\left(-k^{2}+k_{n}^{2}\right)} \\
& \times\left(\begin{array}{c}
e^{i k z}-B_{r} e^{i k_{n} z} \\
e^{i k z}-B_{\theta} e^{i k_{n} z}
\end{array}\right) .
\end{aligned}
$$

The first term in Eq. (8a) is the particular solution of Eq. (6) due to the source term on the right-hand side. The second term is the solution of the homogeneous part of Eq. (6); the coefficients $B_{r}$ and $B_{\theta}$ must be chosen to satisfy the boundary condition, $\left.E_{r}\right|_{z=0}=0$ and $\left.\frac{\partial}{\partial z} H_{\theta}\right|_{z=0}=0$. These conditions can be satisfied by choosing

$$
B_{r}=1 \quad \text { and } \quad B_{\theta}=\frac{k}{k_{n}} .
$$

One can write the denominator of Eq. (8a) in the form

$$
\begin{aligned}
-k^{2}+k_{n}^{2} & =-k^{2}+\left(\varepsilon \mu \frac{\omega^{2}}{c^{2}}-k_{\perp n}^{2}\right) \\
& =\left(\varepsilon \mu \beta^{2}-1\right)\left(k^{2}-k_{0 n}^{2}\right),
\end{aligned}
$$

where

$$
k_{\perp n}^{2} \equiv\left(\varepsilon \mu \beta^{2}-1\right) k_{0 n}^{2} .
$$

By inserting Eq. (8a) back into Eq. (3) and using Eq. (5a), one obtains the fields as

$$
\begin{aligned}
\left(\begin{array}{c}
E_{r}(r, z, t) \\
H_{\theta}(r, z, t)
\end{array}\right)= & 2 q_{b}\left(\begin{array}{c}
1 \\
\varepsilon \beta
\end{array}\right) \sum_{n=1}^{\infty} \frac{e_{z n}\left(r_{0}\right) e_{z n}^{\prime}(r) k_{0 n}^{2}}{C_{n} k_{\perp n}^{4}} \frac{1}{2 \pi} \\
& \times \int_{-\infty}^{\infty} d k e^{-i k z_{b}} \frac{1}{k^{2}-k_{0 n}^{2}} \\
& \times\left(\begin{array}{c}
e^{i k z}-e^{i k_{n} z} \\
e^{i k z}-\frac{k}{k_{n}} e^{i k_{n} z}
\end{array}\right) \\
\left(\begin{array}{c}
E_{r}(r, z, t) \\
H_{\theta}(r, z, t)
\end{array}\right)= & -\frac{E_{0}}{\sqrt{\varepsilon \mu \beta^{2}-1}}\left(\begin{array}{c}
1 \\
\varepsilon \beta
\end{array}\right) \sum_{n=1}^{\infty} \Gamma_{1 n}(r) \\
& \times\left(\begin{array}{c}
G_{r n}(z, t) \\
G_{\theta n}(z, t)
\end{array}\right),
\end{aligned}
$$

where

$$
\begin{gathered}
G_{r n}(z, t)=k_{0 n}\left[I_{1}-\frac{\partial I_{2}}{\partial z}\right], \\
G_{\theta n}(z, t)=k_{0 n}\left[I_{1}+\frac{\partial I_{2}}{\partial z_{b}}\right], \\
E_{0} \equiv \frac{4 q_{b}}{\varepsilon R_{1}^{2}}, \quad \Gamma_{1 n}(r) \equiv \frac{J_{0}\left(k_{\perp n} r_{b}\right)}{J_{1}^{2}\left(k_{\perp n} R_{1}\right)} J_{1}\left(k_{\perp n} r\right),
\end{gathered}
$$

and

$$
\begin{aligned}
& I_{1} \equiv \frac{1}{2 \pi} \int_{-\infty}^{\infty} d k \frac{e^{i k\left(z-z_{b}\right)}}{k^{2}-k_{0 n}^{2}} \\
& I_{2} \equiv \frac{1}{2 \pi} \int_{-\infty}^{\infty} \frac{d k}{i k_{n}} \frac{e^{i k_{n} z-i k z_{b}}}{\left(k^{2}-k_{0 n}^{2}\right)} .
\end{aligned}
$$

The longitudinal electric field $E_{z}$ can be obtained from Eq. (10a) using the Maxwell equation

$$
\frac{\partial E_{z}}{\partial r}=\frac{\partial E_{r}}{\partial z}+\frac{\mu}{c} \frac{\partial H_{\theta}}{\partial t} .
$$

The result can be written as 


$$
E_{z}=-E_{0} \sum_{n=1}^{\infty} \Gamma_{0 n}(r) G_{z n}(z, t),
$$

where

$$
G_{z n}(z, t)=\left[\frac{\partial}{\partial z} I_{1}+k_{0 n}^{2} I_{2}\right]
$$

and

$$
\Gamma_{0 n}(r) \equiv \frac{J_{0}\left(k_{\perp n} r_{b}\right)}{J_{1}^{2}\left(k_{\perp n} R_{1}\right)} J_{0}\left(k_{\perp n} r\right) .
$$

The integral $I_{1}$ represents the particular solution of Eq. (8a) showing the source effect. In an infinitely long waveguide, this is the only term in the solution. The integral $I_{2}$ originates from the solution of the homogeneous equation (8a) which is required to satisfy the conducting boundary condition, $E_{r}=0$ and $\frac{\partial}{\partial z} H_{\theta}=0$, at the input end $(z=0)$. This term represents the transient part of the solution due to the presence of the boundary at the input end.

\section{A. Calculation of the integral $\boldsymbol{I}_{\mathbf{1}}$}

The integral $I_{1}$ represents the Cerenkov radiation in an infinite waveguide and can be solved using the usual contour integration [1]. In the complex $k$ space, the integral path along the real $k$ is slightly shifted upward to avoid the pair of poles at $k= \pm k_{0 n}$ (Fig. 1).

If $z>z_{b}$, where $z_{b} \equiv \nu t$ is the current position of the beam, the path can be closed by including a half circle with infinite radius in the upper plane. Then the integral is zero because it contains no poles:

$$
I_{1}=0 \quad\left(z>z_{b}\right)
$$

If $0<z<z_{b}$, the path can be closed in the lower plane and the integral is given by the residue theorem from both of the poles at $k= \pm k_{0 n}$. The result is

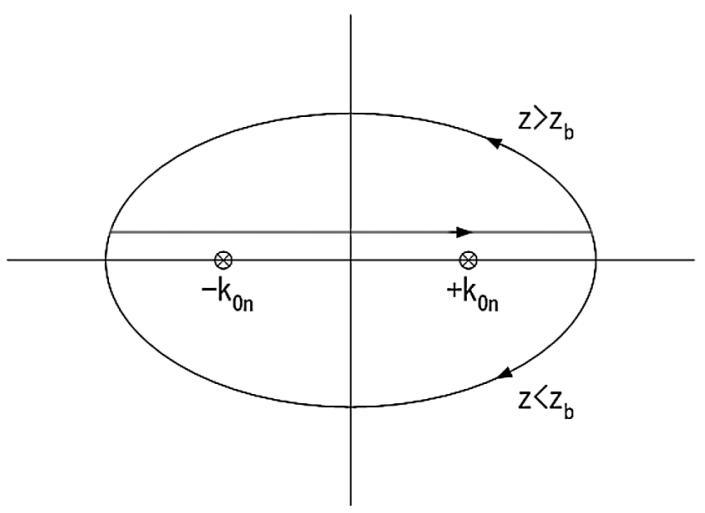

FIG. 1. Contours for integration in the complex $k$ plane for Cerenkov radiation.

$$
\begin{aligned}
I_{1} & =(-2 \pi i) \frac{1}{2 \pi}\left[\frac{e^{i k_{0 n}\left(z-z_{b}\right)}}{2 k_{0 n}}+\frac{e^{-i k_{0 n}\left(z-z_{b}\right)}}{-2 k_{0 n}}\right] \\
& =\frac{1}{k_{0 n}} \sin k_{0 n}\left(z-z_{b}\right) \quad\left(0<z<z_{b}\right) .
\end{aligned}
$$

\section{B. Calculation of the integral $\boldsymbol{I}_{\mathbf{2}}$}

We now consider the integral $I_{2}$ which describes the boundary effect. Compared to $I_{1}$, calculating the integral $I_{2}$ is more difficult, because the integrand contains a branch cut in addition to the same pair of the poles at $k= \pm k_{0 n}$. The branch cut is from the function

$$
k_{n} \equiv \sqrt{\varepsilon \mu \frac{\omega^{2}}{c^{2}}-k_{\perp n}^{2}}=\sqrt{\varepsilon \mu \beta^{2}} \sqrt{k^{2}-\alpha_{n}^{2}}
$$

and it can be conveniently placed on the segment of the real $k$ axis between $k= \pm \alpha_{n}$, where

$$
\alpha_{n}^{2} \equiv \frac{k_{\perp n}^{2}}{\varepsilon \mu \beta^{2}} .
$$

To avoid these singularities, we move the line of integration slightly upward (Fig. 2). Consider the phase $\Psi$ of the exponential function appearing in $I_{2}$ given by Eq. (11):

$$
\Psi \equiv k_{n} z-k z_{b} \equiv-z_{b}\left(k-s \sqrt{k^{2}-\alpha_{n}^{2}}\right),
$$

where

$$
\begin{gathered}
\varsigma \equiv \frac{z}{z_{b}} \sqrt{\varepsilon \mu \beta^{2}}=\frac{z}{z_{p}}, \\
z_{b} \equiv \nu_{b} t, \quad z_{p} \equiv \nu_{p} t, \quad \text { and } \quad \nu_{p} \equiv \frac{c}{\sqrt{\varepsilon \mu}} .
\end{gathered}
$$

In the limit $|k| \rightarrow \infty$, the phase $\Psi \rightarrow-z_{b}(1-\varsigma) k$ and thus the integration path can be closed by including a half circle with infinite radius, (i) in the upper half plane if $s \geq 1$ (i.e. $z \geq z_{p}$ ), and (ii) in the lower half plane if $0<s<1$ (i.e. $0<z<z_{p}$ ). If $1<$ s (i.e. $z_{p}<z$ ), one can close the inte-

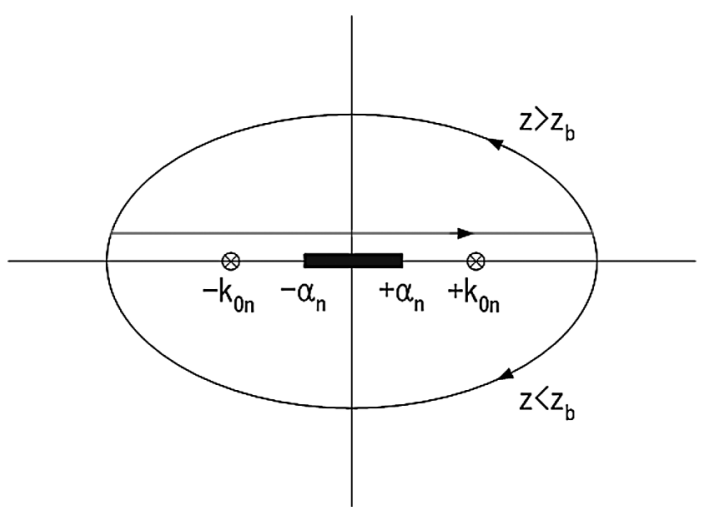

FIG. 2. Contours for integration in the complex $k$ plane for transient radiation. 
gration path in the upper half plane and then $I_{2}$ contains no poles or branch cuts in the upper half plane; thus,

$$
I_{2}=0 \quad\left(z_{p}<z\right) .
$$

This result shows that the signal due to the input boundary effect propagates at a maximum speed of $\nu_{p} \equiv \frac{c}{\sqrt{\varepsilon \mu}}$, i.e., in the region $z_{p}<z<z_{b}$, only Cerenkov radiation due to the source occurs, and no transient radiation has reached the region yet. The Cerenkov radiation part is the same as that in an infinitely long waveguide.

For $0<s<1$ (i.e. $0<z<z_{p}$ ), one can close the integration path in the lower half plane and then $I_{2}$ contains both the branch cut and the poles. We will show that the integral $I_{2}$ is composed of two parts: one is the quenching wave to cancel the Cerenkov radiation and the other is the transient radiation.

This integration $I_{2}$ can be done most easily by performing the conformal mapping suggested in Ref. [13] as

$$
k=\frac{\alpha_{n}}{\sqrt{1-\varsigma^{2}}}(\cos \phi-i \varsigma \sin \phi) .
$$

This function maps the integral path into an ellipse with foci at $\pm \alpha_{n}$ (Fig. 3). In the limit $\varsigma \rightarrow 1$, the ellipse becomes infinitely large, and, in the limit $\varsigma \rightarrow 0$, the ellipse collapses to an infinitely thin one surrounding the branch cut. In the range $0<\varsigma<1$, a point $\varsigma=\varsigma_{q}$ exists at which the ellipse crosses the poles at $k= \pm k_{0 n}$. Thus, $\varsigma_{q}$ is defined by the relation

$$
\left.k\right|_{\phi=0} \equiv \frac{\alpha_{n}}{\sqrt{1-\varsigma_{q}^{2}}}=k_{0 n} .
$$

The meaning of the variable $s_{q}$ is clear if one notices that it corresponds to a point $z=z_{q}$, where

$$
z_{q} \equiv \nu_{q} t \quad \text { and } \quad \nu_{q} \equiv \frac{\nu_{p}^{2}}{\nu_{b}} .
$$

From the definition Eq. (5b), the variable $s$ is proportional to $z$ and the points $s=\left(0, s_{q}, 1\right)$ correspond to the points $z=\left(0, z_{q}, z_{p}\right)$.

The geometrical meaning is clear when the wake is unfolded in the radial direction (Fig. 5).

The integration path now follows this ellipse in the clockwise direction as $\phi$ varies from 0 to $2 \pi$. Let us call this integral $I_{\text {ellipse. }}$. If $\mathrm{s}_{q}<\mathrm{s}<1$,

$$
I_{2}=I_{\text {ellipse }} \quad\left(z_{q}<z<z_{p}\right) .
$$

However, if $0<\mathrm{s}<\mathrm{s}_{q}$,

$$
I_{2}=I_{\text {ellipse }}+I_{\text {poles }} \quad\left(0<z<z_{q}\right),
$$

where $I_{\text {poles }}$ is the contribution from the poles in $I_{2}$. The integral $I_{\text {poles }}$ can be calculated in the same manner as $I_{1}$ by using the residue theorem. However, the branch of the double-valued function $k_{n}$ must be chosen such that the
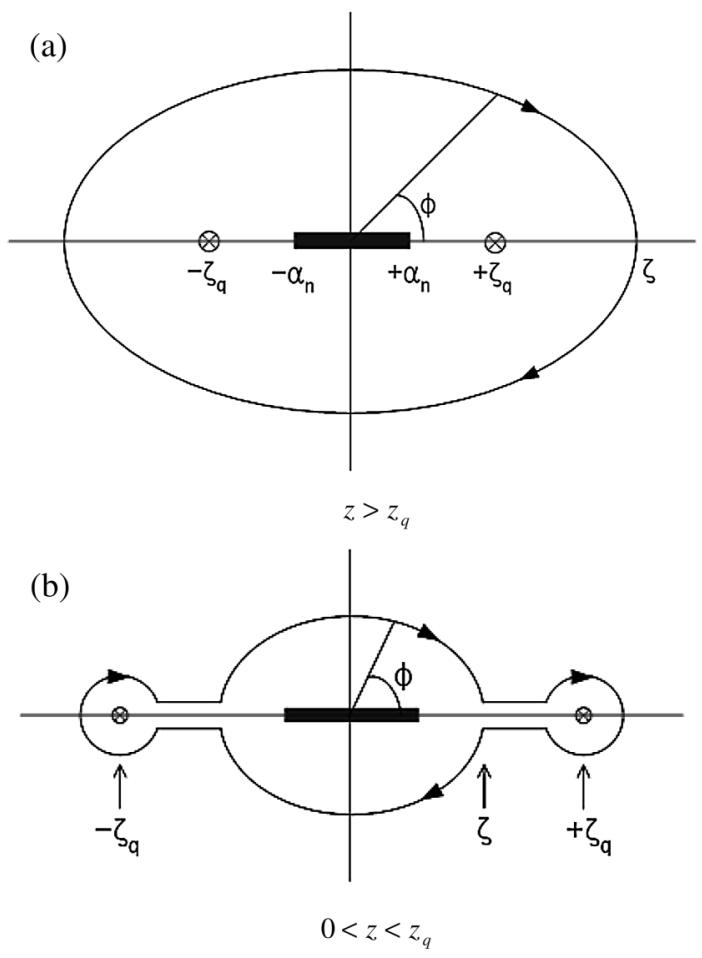

FIG. 3. Integration paths for evaluating transient radiation.

sign of the real part of $k_{n}$ is the same as that of $k$. This choice of the branch guarantees that we are dealing with only the right propagating waves. Then

$$
\begin{aligned}
I_{\text {pole }}= & (-2 \pi i) \frac{1}{2 \pi}\left[\left.\frac{1}{i k_{n}} \frac{e^{\left.i k_{n} z-i k z_{b}\right)}}{k+k_{0 n}}\right|_{k=k_{0 n}}\right. \\
& \left.+\left.\frac{1}{i k_{n}} \frac{e^{\left.i k_{n} z-i k z_{b}\right)}}{k-k_{0 n}}\right|_{k=-k_{0 n}}\right] \\
= & -\left[\frac{1}{k_{0 n}} \frac{e^{i k_{0 n}\left(z-z_{b}\right)}}{2 k_{0 n}}+\frac{1}{-k_{0 n}} \frac{e^{-i k_{0 n}\left(z-z_{b}\right)}}{-2 k_{0 n}}\right] \\
= & \frac{-1}{k_{0 n}^{2}} \cos k_{0 n}\left(z-z_{b}\right),
\end{aligned}
$$

where we have used the fact that

$$
\left.k_{n}\right|_{k= \pm k_{0 n}}= \pm\left.\sqrt{\varepsilon \mu \beta^{2} k^{2}-k_{\perp n}^{2}}\right|_{k= \pm k_{0 n}}= \pm k_{0 n} .
$$

Notice that $I_{\text {pole }}$ given by Eq. (20), i.e. the pole contribution part of $I_{2}$, exactly cancels $I_{1}$ given by Eq. (14b). This cancellation in $E_{z}$ can be easily seen from Eqs. (13a), (14b), and (20). Also the cancellation in $E_{r}$ and $H_{\theta}$ can be checked from Eqs. (10a), (14b), and (20). For this reason, this term is sometimes called the "quench wave" [15]. Therefore, as far as the Cerenkov radiation is concerned, the only effect of the input boundary is to terminate the Cerenkov radiation after $z_{q}$. As a result, the Cerenkov radiation in the presence of the quench wave does not vanish only in the range $z_{q}<z<z_{b}$ and the fields are given by 


$$
\begin{aligned}
E_{z}^{(c)}(r, z, t)= & -E_{0} \sum_{n=1}^{\infty} \Gamma_{0 n}(r) \cos k_{0 n}\left(z-z_{b}\right) \\
\left(\begin{array}{c}
E_{r}^{(c)}(r, z, t) \\
H_{r}^{(c)}(r, z, t)
\end{array}\right)= & -\frac{E_{0}}{\sqrt{\varepsilon \mu \beta^{2}-1}}\left(\begin{array}{c}
1 \\
\varepsilon \beta
\end{array}\right) \\
& \times \sum_{n=1}^{\infty} \Gamma_{1 n}(r) \sin k_{0 n}\left(z-z_{b}\right) .
\end{aligned}
$$

\section{Calculation of the integral $I_{\text {ellipse }}$}

Now, we consider the transient radiation part of $I_{2}$ given by $I_{\text {ellipse }}$. In terms of the variable $\phi$ defined by Eq. (18a), the phase function $\Psi$ can be written as

$$
\Psi=-Z_{n} \cos \phi
$$

where

$$
Z_{n} \equiv z_{b} \alpha_{n} \sqrt{1-\varsigma^{2}}=\left|k_{\perp n}\right| \sqrt{z_{p}^{2}-z^{2}} .
$$

We introduce a new variable $\chi$ such that

$$
k=\frac{\alpha_{n}}{2}\left(\chi+\frac{1}{\chi}\right)
$$

and write $\chi$ as

$$
\chi \equiv \xi e^{-i \phi},
$$

where

$$
\xi \equiv \sqrt{\frac{1+\varsigma}{1-\varsigma}}=\sqrt{\frac{z_{p}+z}{z_{p}-z}}
$$

In terms of this new variable, it is straightforward to show that

$$
\frac{d k}{i k_{n}}=\frac{1}{\sqrt{\varepsilon \mu \beta^{2}}} \frac{d \chi}{i \chi}=\frac{-d \phi}{\sqrt{\varepsilon \mu \beta^{2}}}
$$

and

$$
\begin{aligned}
\frac{1}{k^{2}-k_{0 n}^{2}} & =\frac{1}{2 k_{0 n}}\left(\frac{1}{k-k_{0 n}}-\frac{1}{k+k_{0 n}}\right) \\
& =\frac{\sqrt{\varepsilon \mu \beta^{2}}}{k_{0 n}^{2}}\left(\frac{\xi_{q}^{2}}{\chi^{2}-\xi_{q}^{2}}-\frac{1}{\chi^{2} \xi_{q}^{2}-1}\right)
\end{aligned}
$$

where

$$
\xi_{q} \equiv \frac{k_{0 n}}{\alpha_{n}}+\sqrt{\frac{k_{0 n}^{2}}{\alpha_{n}^{2}}-1}=\sqrt{\frac{z_{p}+z_{q}}{z_{p}-z_{q}}}
$$

Equation (24b) can be expanded in a power series and its convergence is guaranteed by comparing the relative magnitudes of $\chi$ and $\xi_{q}$ in the region of interest $0<s<1$. In the region $\mathrm{s}_{q}<\mathrm{s}<1$, we find $\xi_{q}<\xi$, and thus one can expand the first term of Eq. (24a) in the power of $\left(\frac{\xi_{q}}{\chi}\right)^{2}$. In the region $0<\mathrm{s}<\boldsymbol{s}_{q}$, one can show that $\xi<\xi_{q}$ and thus one can expand the first term of Eq. (24a) in the power of $\left(\frac{\chi}{\xi_{q}}\right)^{2}$. One can expand the second term of Eq. (24a) in the power of $\left(\frac{1}{\chi \xi_{q}}\right)^{2}$ in the entire region of $0<\xi<1$, by noticing that $1<\xi$ and $1<\xi_{q}$. Then we obtain

$$
\frac{1}{k^{2}-k_{0 n}^{2}}=\frac{\sqrt{\varepsilon \mu \beta^{2}}}{k_{0 n}^{2}} \begin{cases}\sum_{m=1}^{\infty}\left(\frac{\xi_{q}}{\chi}\right)^{2 m}-\sum_{m=1}^{\infty}\left(\frac{1}{\chi \xi_{q}}\right)^{2 m} & \left(z_{q}<z<z_{p}\right) \\ -\sum_{m=0}^{\infty}\left(\frac{\chi}{\xi_{q}}\right)^{2 m}-\sum_{m=1}^{\infty}\left(\frac{1}{\chi \xi_{q}}\right)^{2 m} & \left(0<z<z_{q}\right) .\end{cases}
$$

Using Eqs. (23d) and (25), one can write the integration $I_{\text {ellipse }}$ as

$$
I_{\text {ellipse }}=\frac{1}{k_{0 n}^{2}} \frac{1}{2 \pi} \int_{0}^{2 \pi} d \phi e^{-i Z_{n} \cos \phi} \begin{cases}\sum_{m=1}^{\infty} e^{i 2 m \phi}\left(\left(\frac{1}{\xi \xi_{q}}\right)^{2 m}-\left(\frac{\xi_{q}}{\xi}\right)^{2 m}\right) & \left(z_{q}<z<z_{p}\right) \\ 1+\sum_{m=1}^{\infty}\left(e^{i 2 m \phi}\left(\frac{1}{\xi \xi_{q}}\right)^{2 m}+e^{-i 2 m \phi}\left(\frac{\xi}{\xi_{q}}\right)^{2 m}\right) & \left(0<z<z_{q}\right) .\end{cases}
$$

This integration can be done immediately in terms of Bessel functions by recalling the relation

$$
J_{m}\left(Z_{n}\right)=\frac{i^{m}}{2 \pi} \int_{0}^{2 \pi} d \phi e^{-i Z_{n} \cos \phi \pm i m \phi} .
$$

The result can be written as

$$
I_{\text {ellipse }}=\frac{1}{k_{0 n}^{2}} \begin{cases}U_{0}\left(\frac{1}{\xi_{q} \xi} Z_{n}, Z_{n}\right)-U_{0}\left(\frac{\xi_{q}}{\xi} Z_{n}, Z_{n}\right) & \left(z_{q}<z<z_{p}\right) \\ -J_{0}\left(Z_{n}\right)+U_{0}\left(\frac{1}{\xi_{q} \xi} Z_{n}, Z_{n}\right)+U_{0}\left(\frac{\xi}{\xi_{q}} Z_{n}, Z_{n}\right) & \left(0<z<z_{q}\right),\end{cases}
$$


where

$$
U_{n}(w, x) \equiv \sum_{m=0}^{\infty}(-1)^{m}\left(\frac{w}{x}\right)^{n+2 m} J_{n+2 m}(x)
$$

is the Lommel function [16] and

$$
\begin{aligned}
Z_{n} & \equiv\left|k_{\perp n}\right| \sqrt{\left(z_{p}+z\right)\left(z_{p}-z\right)} \\
\xi & \equiv \sqrt{\frac{z_{p}+z}{z_{p}-z}} \quad\left(z_{p} \equiv \nu_{p} t\right) .
\end{aligned}
$$

The longitudinal field $E_{z}$ can now be obtained from Eqs. (13a)-(13c):

$$
E_{z}^{(t)}(r, z, t)=-E_{0} \sum_{n=1}^{\infty} \Gamma_{0 n}(r) G_{z n t z n}^{(t)}(z, t),
$$

where

$$
G_{z n}^{(t)}(z, t)= \begin{cases}U_{0}\left(\frac{1}{\xi_{q} \xi} Z_{n}, Z_{n}\right)-U_{0}\left(\frac{\xi_{q}}{\xi} Z_{n}, Z_{n}\right) & \left(z_{q}<z<z_{p}\right) \\ -J_{0}\left(Z_{n}\right)+U_{0}\left(\frac{1}{\xi_{q} \xi} Z_{n}, Z_{n}\right)+U_{0}\left(\frac{\xi}{\xi_{q}} Z_{n}, Z_{n}\right) & \left(0<z<z_{q}\right) .\end{cases}
$$

The transverse components of the field can now be easily calculated from Eq. (21) with the help of the above relations. The results can be summarized as

$$
E_{r}^{(t)}(r, z, t)=-\frac{E_{0}}{\sqrt{\varepsilon \mu \beta^{2}-1}} \sum_{n=1}^{\infty} \Gamma_{1 n}(r) G_{r n}^{(t)}(z, t)
$$

where

$$
G_{r n}^{(t)}(z, t)= \begin{cases}U_{1}\left(\frac{1}{\xi_{q} \xi} Z_{n}, Z_{n}\right)+U_{1}\left(\frac{\xi_{q}}{\xi} Z_{n}, Z_{n}\right) & \left(z_{q}<z<z_{p}\right) \\ U_{1}\left(\frac{1}{\xi_{q} \xi} Z_{n}, Z_{n}\right)-U_{1}\left(\frac{\xi}{\xi_{q}} Z_{n}, Z_{n}\right) & \left(0<z<z_{q}\right),\end{cases}
$$

and

$$
\begin{aligned}
& H_{\theta}^{(t)}(r, z, t)=-\frac{E_{0} \varepsilon \beta}{\sqrt{\varepsilon \mu \beta^{2}-1}} \sum_{n=1}^{\infty} \Gamma_{1 n}(r) G_{\theta n}^{(t)}(z, t) \\
& G_{\theta n}^{(t)}(z, t)= \begin{cases}-U_{1}\left(\frac{1}{\xi_{q} \xi} Z_{n}, Z_{n}\right)+U_{1}\left(\frac{\xi_{q}}{\xi} Z_{n}, Z_{n}\right) & \left(z_{q}<z<z_{p}\right) \\
U_{1}\left(\frac{1}{\xi_{q} \xi} Z_{n}, Z_{n}\right)-U_{1}\left(\frac{\xi}{\xi_{q}} Z_{n}, Z_{n}\right) & \left(0<z<z_{q}\right) .\end{cases}
\end{aligned}
$$

The electric flux lines in the wakefield (Fig. 4) were computed using the solution with the Runge-Kutta method when a beam flights $2.16 \mathrm{~cm}$ in a semi-infinite waveguide having a radius of $R=0.5 \mathrm{~cm}$ filled with a dielectric of $\varepsilon=3$, and a $2 \mathrm{nC}, 30.7 \mathrm{MeV}(\gamma=61)$ beam bunch moving uniformly along the axis. Because the wakefield from a point charge diverges, the bunch is assumed to have a radial distribution described by a Bessel function with a radius of $\Delta r_{b}=0.05 \mathrm{~cm}$.

The lineal charge density $\Sigma(r, z)$ in Fig. 4 is related to the electric flux density $\Phi_{w}(r, z)$ on the wall through Gauss's law as $\Phi_{w}(r, z)=2 \pi r E_{\perp}(r, z)=4 \pi \Sigma(r, z) / \varepsilon$. By integrating the surface charge on the whole conducting boundary, the charge induced on the wall of the cavity is found to be $-2 \mathrm{nC}$, which is exactly the same with the bunch charge with opposite sign.

Figure 5 shows electric field lines of the wakefield. Red and blue lines represent Cerenkov and transient radiation, respectively. A Cerenkov cone ends up at the quenching point $\left(z_{q}\right)$ because fields, where $z<z_{q}$, cannot pass
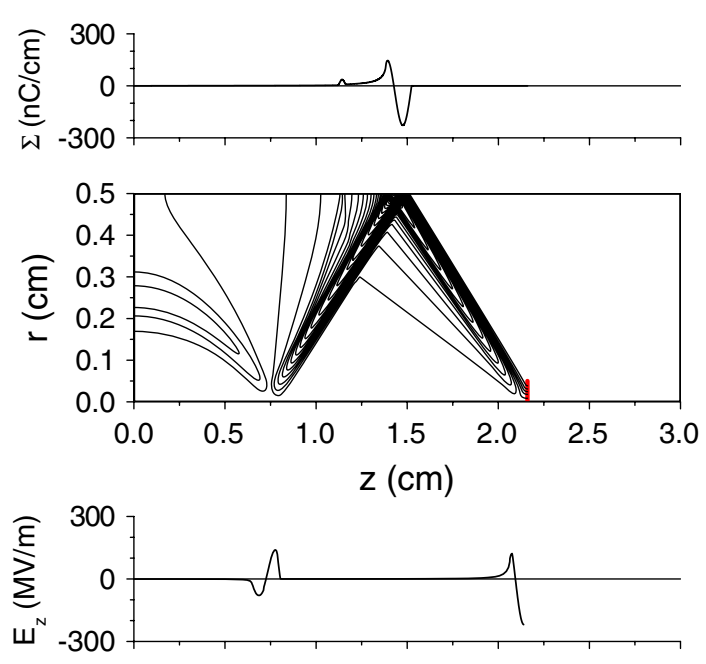

FIG. 4. Flux lines, axial electric field on the beam axis $\left(E_{Z}\right)$, and the lineal surface charge density $(\Sigma)$ of a wakefield in a semi-infinite waveguide filled with a dielectric. 

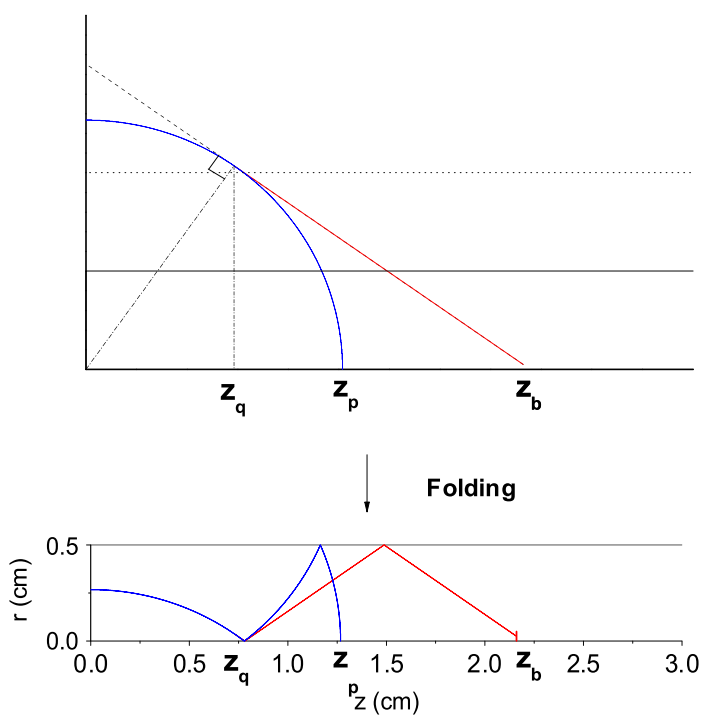

FIG. 5. (Color) Electric field lines of the wakefield when a beam passes through $z=z_{b}$ (red: Cerenkov radiation; blue: transient radiation). Radiation lines in a semi-infinite waveguide (below) can be obtained by folding lines in a space without radial boundary (above) on the radial boundary.

through a transient spherical shell. Surfaces of wakefield in a cylindrical waveguide can be obtained with folding surfaces on a conducting boundary. The flux line (Fig. 4) using the analytic solution agrees exactly with lines of radiation (Fig. 5).

\section{WAKEFIELD WITH OUTPUT BOUNDARY}

To construct wakefields in a cavity, one must solve the effect of the boundary at the output end. Assume that the boundary is a thin conducting foil which allows a high energy beam to pass through freely, but reflects the wakefield. The boundary condition at the output end for each charge can be decomposed into two conditions: (1) the charge inside the cavity becomes zero when the charge $q_{b}$ reached output end and (2) the wakefield is reflected by the boundary wall.

The first boundary condition is satisfied when the charge $q_{b}$ has reached the output end and at the same time that the anticharge $\bar{q}_{b}\left(=-q_{b}\right)$ has started at the output end: when $\bar{q}_{b}$, which is of equal magnitude to $q_{b}$ but opposite sign, started the output end with charge $q_{b}$ at the same time instead of disappearing charge $q_{b}$ at the output end, total charge becomes zero. Therefore, the first boundary condition is satisfied.

The solution of the wakefield induced by $\bar{q}_{b}$ can apply to the wakefield solution of a semi-infinite waveguide, but it must take into account the boundary condition which considers the output end to be the input wall of a semi-infinite waveguide.

To calculate the field from the anticharge, it is convenient to use the transformed coordinate $\bar{z}_{b}=z_{b}-d$; then the $E_{z}$ field induced by the anticharge can be obtained similarly to Eqs. (21) and (29a):

$$
\begin{aligned}
& \bar{E}_{c z}(r, \bar{z}, \bar{t})=E_{0} \sum_{n=1}^{\infty} \Gamma_{0 n}(r) \cos k_{0 n}\left(\bar{z}-\bar{z}_{b}\right) \\
& \bar{E}_{t z}(r, \bar{z}, \bar{t})=E_{0} \sum_{n=1}^{\infty} \Gamma_{0 n}(r) \bar{G}_{t z n}(\bar{z}, \bar{t})
\end{aligned}
$$

where

$$
\bar{G}_{t r n}(\bar{z}, \bar{t})= \begin{cases}+U_{1}\left(\frac{1}{\bar{\xi}_{q} \bar{\xi}} \bar{Z}_{n}, \bar{Z}_{n}\right)+U_{1}\left(\frac{\bar{\xi}_{q}}{\bar{\xi}} \bar{Z}_{n}, \bar{Z}_{n}\right) & \left(\bar{z}_{q}<\bar{z}<\bar{z}_{p}\right) \\ +U_{1}\left(\frac{1}{\bar{\xi}_{q} \bar{\xi}} \bar{Z}_{n}, \bar{Z}_{n}\right)-U_{1}\left(\frac{\bar{\xi}_{q}}{\bar{\xi}} \bar{Z}_{n}, \bar{Z}_{n}\right) & \left(0<\bar{z}<\bar{z}_{q}\right)\end{cases}
$$

where the plus sign of the field is expressed to emphasize that the anticharge and the real charge signs are opposite each other. Similarly, the other field can be obtained easily by coordinate transformation (Fig. 7).

Folding the field (Fig. 5) satisfies all of the boundary conditions imposed by the conducting wall. One can fold the field $l$ times with the simple coordinate transform as

$$
\begin{aligned}
& z_{l}^{\prime} \equiv \begin{cases}z+l d & (l=\text { even }) \\
(d-z)+l d & (l=\text { odd })\end{cases} \\
& \bar{z}_{l}^{\prime} \equiv z_{l}^{\prime}-d,
\end{aligned}
$$

where the primed coordinate represents the semi-infinite waveguide and the unprimed coordinate represents the cavity.
We now consider the boundary condition introduced by the conducing wall, and calculate wakefields of the cavity. The particular solution of the cavity's wakefields is given by

$$
\begin{aligned}
G_{z n}^{(c r)}(z)= & g_{z n}\left(z, z_{q}\right)+\sum_{l=1}^{\infty}\left[g_{z n}\left(z_{l}^{\prime}, z_{q}\right)-g_{z n}\left(\bar{z}_{l}^{\prime}, \bar{z}_{q}\right)\right] \\
G_{r n}^{(c r)}(z)= & g_{\perp n}\left(z, z_{q}\right)+\sum_{l=1}^{\infty}(-1)^{l}\left[g_{\perp n}\left(z_{l}^{\prime}, z_{q}\right)\right. \\
& \left.-g_{\perp n}\left(\bar{z}_{l}^{\prime}, \bar{z}_{q}\right)\right] \\
G_{\theta n}^{(c r)}(z)= & g_{\perp n}\left(z, z_{q}\right)+\sum_{l=1}^{\infty}\left[g_{\perp n}\left(z_{l}^{\prime}, z_{q}\right)-g_{\perp n}\left(\bar{z}_{l}^{\prime}, \bar{z}_{q}\right)\right],
\end{aligned}
$$


where

$$
\begin{aligned}
g_{z n}\left(z, z_{q}\right) & \equiv \cos k_{0 n}\left(z-z_{b}\right) \Theta\left(z_{b}-z\right) \Theta\left(z-z_{q}\right) \\
g_{\perp n}\left(z, z_{q}\right) & \equiv \sin k_{0 n}\left(z-z_{b}\right) \Theta\left(z_{b}-z\right) \Theta\left(z-z_{q}\right),
\end{aligned}
$$

and the transient solution is given by

$$
\begin{aligned}
G_{z n}^{(t r)}(z)= & f_{z n}\left(z, z_{p}, z_{q}\right)+\sum_{l=1}^{\infty}\left[f_{z n}\left(z_{l}^{\prime}, z_{p}, z_{q}\right)\right. \\
& \left.-f_{z n}\left(\bar{z}_{l}^{\prime}, \bar{z}_{p}, \bar{z}_{q}\right)\right] \\
G_{r n}^{(t r)}(z)= & f_{r n}\left(z, z_{p}, z_{q}\right)+\sum_{l=1}^{\infty}(-1)^{l}\left[f_{r n}\left(z_{l}^{\prime}, z_{p}, z_{q}\right)\right. \\
& \left.-f_{r n}\left(\bar{z}_{l}^{\prime}, \bar{z}_{p}, \bar{z}_{q}\right)\right] \\
G_{\theta n}^{(t r)}(z)= & f_{\theta n}\left(z, z_{p}, z_{q}\right)+\sum_{l=1}^{\infty}\left[f_{\theta n}\left(z_{l}^{\prime}, z_{p}, z_{q}\right)\right. \\
& \left.-f_{\theta n}\left(\bar{z}_{l}^{\prime}, \bar{z}_{p}, \bar{z}_{q}\right)\right],
\end{aligned}
$$

where

$$
\begin{aligned}
f_{z n}\left(z, z_{p}, z_{q}\right)= & {\left[U_{0}\left(\frac{1}{\xi_{q} \xi} \chi_{n}, \chi_{n}\right)-U_{0}\left(\frac{\xi_{q}}{\xi} \chi_{n}, \chi_{n}\right)\right] } \\
& \times \Theta\left(z_{p}-z\right) \Theta\left(z-z_{q}\right) \\
& +\left[-J_{0}\left(\chi_{n}\right)+U_{0}\left(\frac{1}{\xi_{q} \xi} \chi_{n}, \chi_{n}\right)\right. \\
& \left.+U_{0}\left(\frac{\xi}{\xi_{q}} \chi_{n}, \chi_{n}\right)\right] \Theta\left(z_{q}-z\right) \Theta(z) \\
f_{r n}\left(z, z_{p}, z_{q}\right)= & {\left[U_{1}\left(\frac{1}{\xi_{q} \xi} \chi_{n}, \chi_{n}\right)+U_{1}\left(\frac{\xi_{q}}{\xi} \chi_{n}, \chi_{n}\right)\right] } \\
& \times \Theta\left(z_{p}-z\right) \Theta\left(z-z_{q}\right) \\
& +\left[U_{1}\left(\frac{1}{\xi_{q} \xi} \chi_{n}, \chi_{n}\right)-U_{1}\left(\frac{\xi}{\xi_{q}} \chi_{n}, \chi_{n}\right)\right] \\
& \times \Theta\left(z_{q}-z\right) \Theta(z) \\
& +\left[-U_{1}\left(\frac{1}{\xi_{q} \xi} \chi_{n}, \chi_{n}\right)-U_{1}\left(\frac{\xi}{\xi_{q}} \chi_{n}, \chi_{n}\right)\right] \\
& \times \Theta\left(z_{q}-z\right) \Theta(z) . \\
f_{\theta n}\left(z, z_{p}, z_{q}\right)= & \left.-U_{1}\left(\frac{1}{\xi_{q} \xi} \chi_{n}, \chi_{n}\right)+U_{1}\left(\frac{\xi_{q}}{\xi} \chi_{n}, \chi_{n}\right)\right] \\
& {[35 \mathrm{c}) } \\
& \\
& \\
&
\end{aligned}
$$

Figure 6 shows the electric flux lines in the wakefield when $z_{b}=2 d$ in a cavity having a length of $d=3 \mathrm{~cm}$ and a radius of $R=0.5 \mathrm{~cm}$ filled with a dielectric of $\varepsilon=3$, and a $2 \mathrm{nC}$ and $30.7 \mathrm{MeV}(\gamma=61)$ beam bunch moving uniformly along the axis. The radius of a beam bunch is $\Delta r_{b}=0.05 \mathrm{~cm}$. Flux lines of radiation are reflected on the
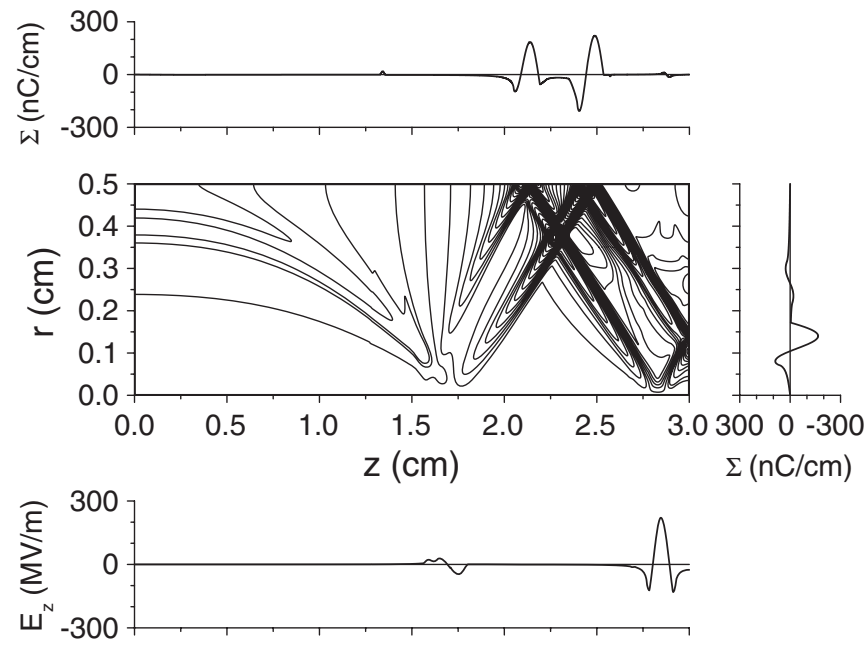

FIG. 6. Flux lines, axial electric field on the beam axis, and the lineal surface charge density of the wakefield in the dielectricfilled cavity when $z_{b}=2 d$.

output boundary. To understand the flux line, we draw and fold electric field lines as shown in Fig. 7.

Figure 7 shows the electric field line in a cavity filled with a dielectric. After drawing an electric field line of Cerenkov and transient with the only boundary of conducting surface on the input plane, we can understand the
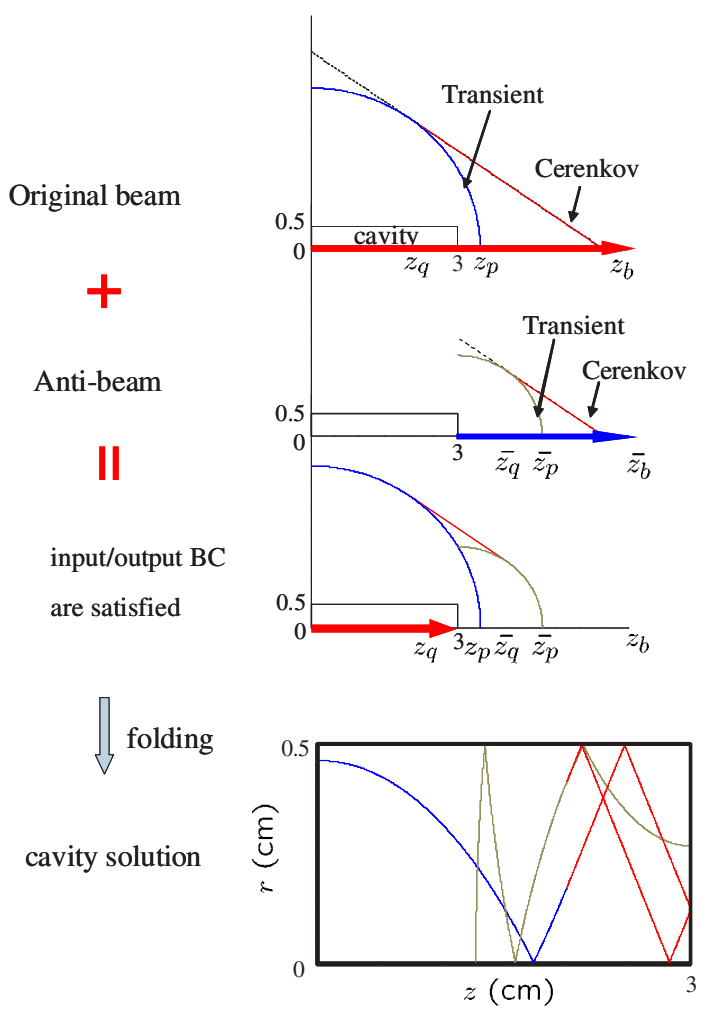

FIG. 7. (Color) Electric field lines in a cavity: Folding Cerenkov and transient line from the original charge and anticharge, we can draw the electric field line in a cavity. 

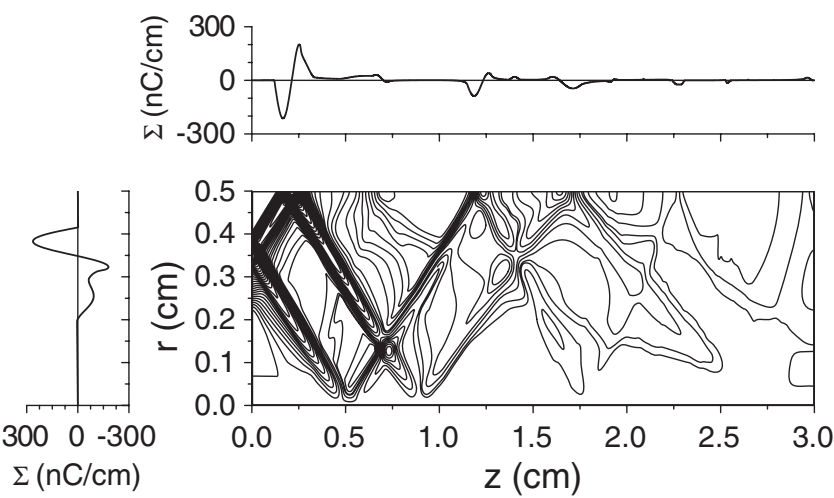

$300 \quad 0-300$

$\Sigma(\mathrm{nC} / \mathrm{cm})$

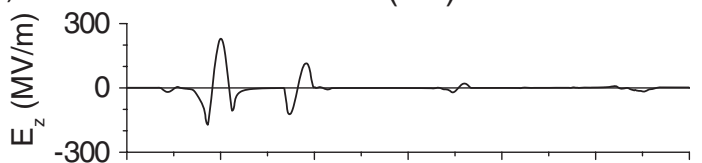

FIG. 8. (Color) Flux lines, axial electric field on the beam axis, and the lineal surface charge density of the wakefield in the dielectric-filled cavity when $z_{b}=5 d$.

electric field line in a cavity by folding lines on conducting boundaries. Because Cerenkov radiation is only affected by the relative location from the charge, the fields induced by the original charge and anticharge, which have the same magnitude but opposite sign, cancel out completely. We now consider the wakefield solution that can be completed by applying the second boundary condition (i.e., the wakefield is reflected by the boundary wall of the cavity).

Figures 6 and 7 describe all the same system. The flux line (Fig. 6) agrees exactly with lines of the electric field (Fig. 7), therefore one can see that the strong flux line represents Cerenkov radiation.

Figures 8 and 9 show flux lines drawn with the same condition as Figs. 6 and 7 except for $z_{b}=5 d$ and $z_{b}=$ $10 d$, respectively. The fields are reflected on input and output boundaries.
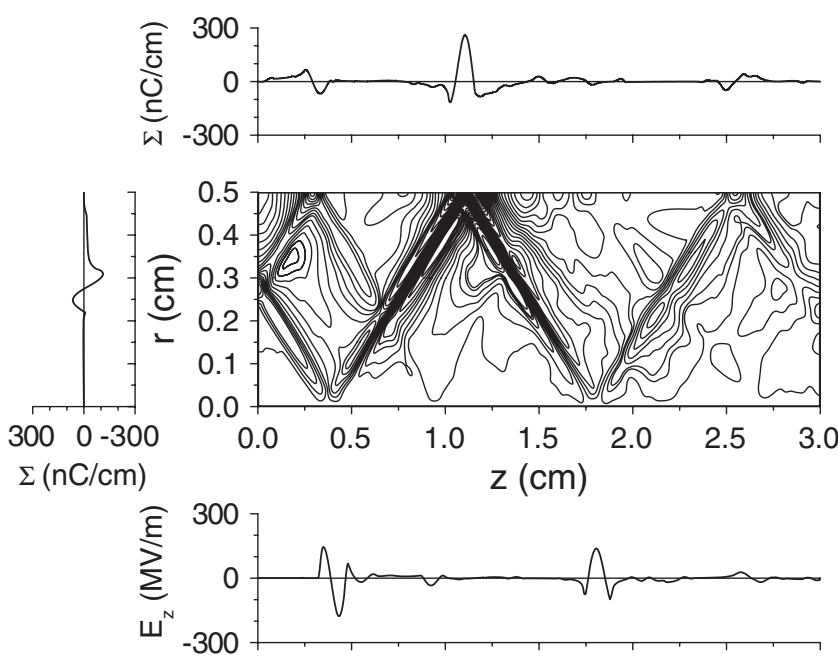

FIG. 9. Flux lines, axial electric field on the beam axis, and the lineal surface charge density of the wakefield in the dielectricfilled cavity when $z_{b}=10 \mathrm{~d}$.
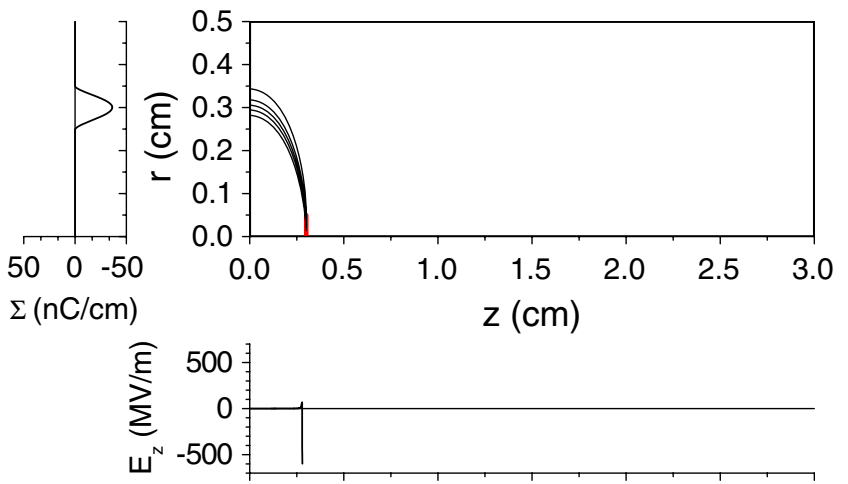

FIG. 10. Flux lines, axial electric field on the beam axis, and the lineal surface charge density, just after a beam bunch passed through the entrance boundary of a cavity filled with dielectric of $\varepsilon=1.1$.

After the beam passes through the cavity, the axial length of a Cerenkov radiation line $\Lambda$ becomes

$$
\Lambda=\bar{z}_{q}-z_{q}+d=\left(1-\frac{1}{\varepsilon \mu \beta^{2}}\right) d,
$$

where all the variables are independent of time or the length of the Cerenkov radiation line remains unchanged.

In order to compare this result with the well-known vacuum solutions [14], we examined the low dielectricconstant limit of our solution. For that purpose a cavity filled with a dielectric of $\varepsilon=1.1$ is assumed. Because the phase velocity of electromagnetic wave in the cavity $\nu_{p}$ is $0.9535 c$, a beam bunch with $\gamma=3.4$ or $\nu_{b}=0.9558 c$, which is similar to but still faster than the phase velocity, was chosen for analysis.

Figure 10 shows flux lines when a beam just entered the cavity $\left(z_{b}=0.3 \mathrm{~cm}\right)$. The feature that most flux lines are
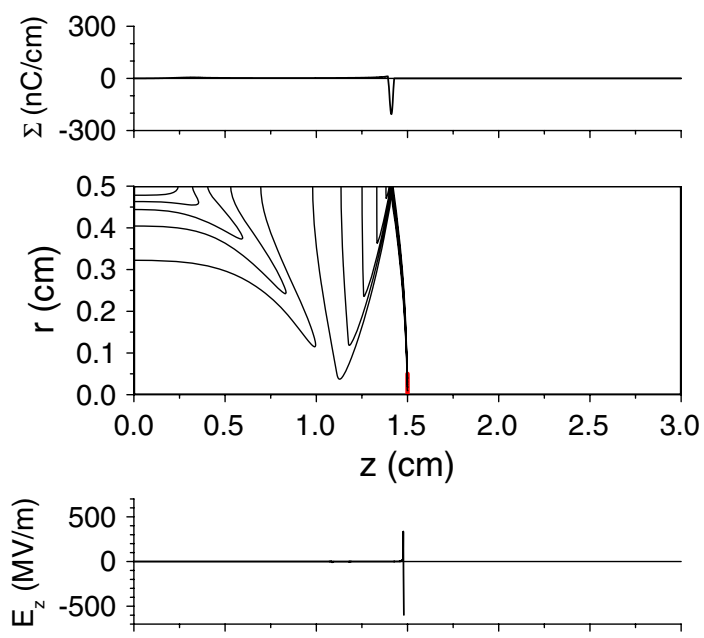

FIG. 11. (Color) Flux lines, axial electric field on the beam axis, and the lineal surface charge density when a beam bunch reaches the middle of the cavity in Fig. 10. 

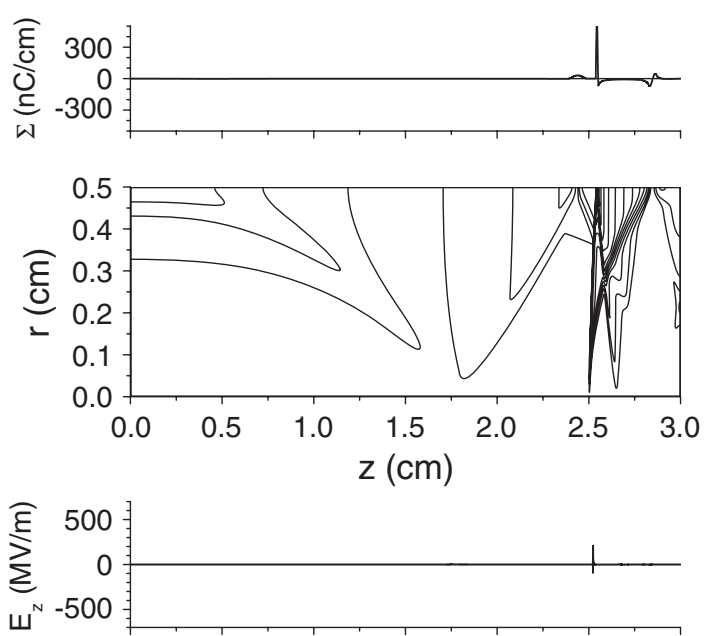

FIG. 12. Flux lines, axial electric field on the beam axis, and the lineal surface charge density, just after a beam bunch passed through the exit boundary of a cavity in Figs. 10 and 11.

concentrated in a thin quasispherical shell containing the beam, agrees with the vacuum solution.

When a beam bunch moves away from the entrance boundary, flux lines stretched out to form a quasiplanar disk as shown in Fig. 11. This feature also agrees well with the vacuum solution. Some wakefield lines appear behind the Coulomb field because the wakefield condition, such that charge moves faster than electromagnetic wave, is still met.

One can easily find that, by unfolding flux lines as previously introduced in Fig. 5, flux lines still form a quasispherical shell. As parameters of cavity and beam bunch approach a vacuum limit, the limit of $\varepsilon \mu \beta^{2} \rightarrow 1$ more generally, the quenching point gets toward the beam position. Therefore, near the vacuum limit, Cerenkov radiation does not exist anymore and the wakefield takes the shape of a spherical shell which is mainly formed by a transient field. As the beam bunch drifts farther from the entrance boundary, the radius of the sphere becomes larger so that flux lines near the beam are shaped like a planar disk.

When a beam bunch leaves the exit boundary of the cavity, another transient field originated by antibeam is added to the wakefield. Therefore, as shown in Fig. 12, flux lines form two spherical shells. With taking account of the method of the folding field in a cavity, this feature corresponds with the wakefield for two infinite parallel plates in vacuum [14].

\section{MULTIPLE BUNCHES}

In a dielectric-lined waveguide, an analytical theory has been developed for the enhancement of acceleration gradients by Park et al. [17] using a train of beam bunches. However, it is unrealistic because of the infinite waveguide.
Therefore, we need to study for a dielectric wakefield accelerator in a more realistic way.

To enhance the acceleration gradient in a dielectricfilled cavity, the resonance condition must be satisfied: (1) cavity resonance condition: Cerenkov radiation from each bunch should be superimposed constructively, and (2) synchronization condition: quenching points of the wakefield from all bunches should be identical in the cavity.

The cavity resonance condition is to match Cerenkov radiation from each bunch. This condition is

$$
d=n \frac{L}{2},
$$

where $n$ is an integer and $L$ is a distance between peaks of the electric field of Cerenkov radiation on the $z$ axis such that

$$
L=2 R \sqrt{\left(\frac{\nu_{b}}{\nu_{p}}\right)^{2}-1}=2 R \sqrt{\varepsilon \mu \beta^{2}-1} .
$$

The synchronization condition means that a following bunch should enter the cavity on time when the head quench point of the former bunch reaches the input boundary of the cavity. The time interval $T$ between adjacent bunches that satisfies this condition is

$$
T=\frac{2 d}{\nu_{q}}=2 d \frac{\varepsilon \mu \beta}{c} .
$$

Figure 13(a) shows the acceleration gradient when $t=$ $0.15 T$, the first bunch is in the cavity. All parameters are the same as those in Figs. 4, 6, 8, and 9 except the length of the cavity, $d$, is tuned to $2.828 \mathrm{~cm}$ which meets the resonance condition. The period of bunches, $T$, is $0.566 \mathrm{~ns}$ which satisfies the synchronization condition. One can easily notice that the plot shows two peaks. The first
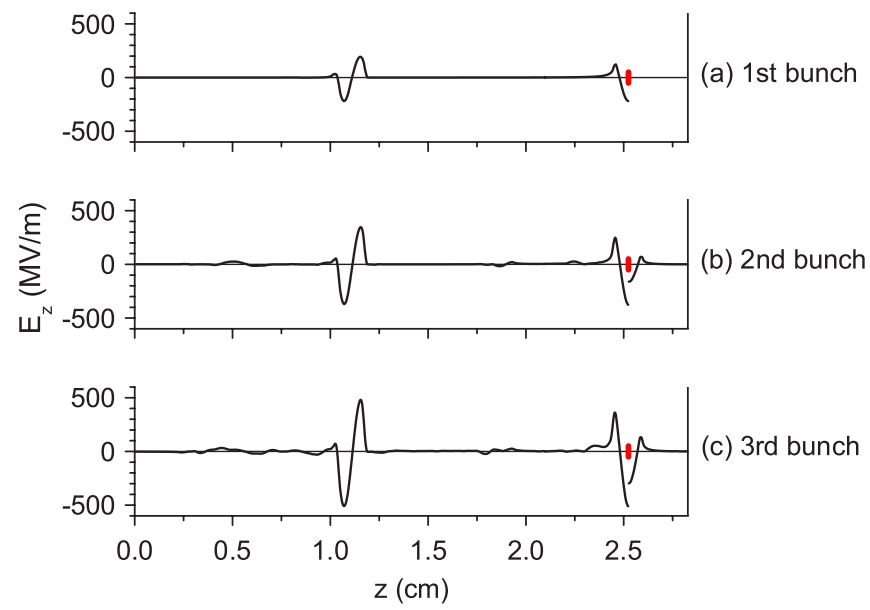

FIG. 13. (Color) Acceleration gradient when each of (a) 1st bunch, (b) 2nd bunch, and (c) 3rd bunch is in the cavity. The field was measured on $r=0.01 R$ because it diverges on the $z$ axis. 

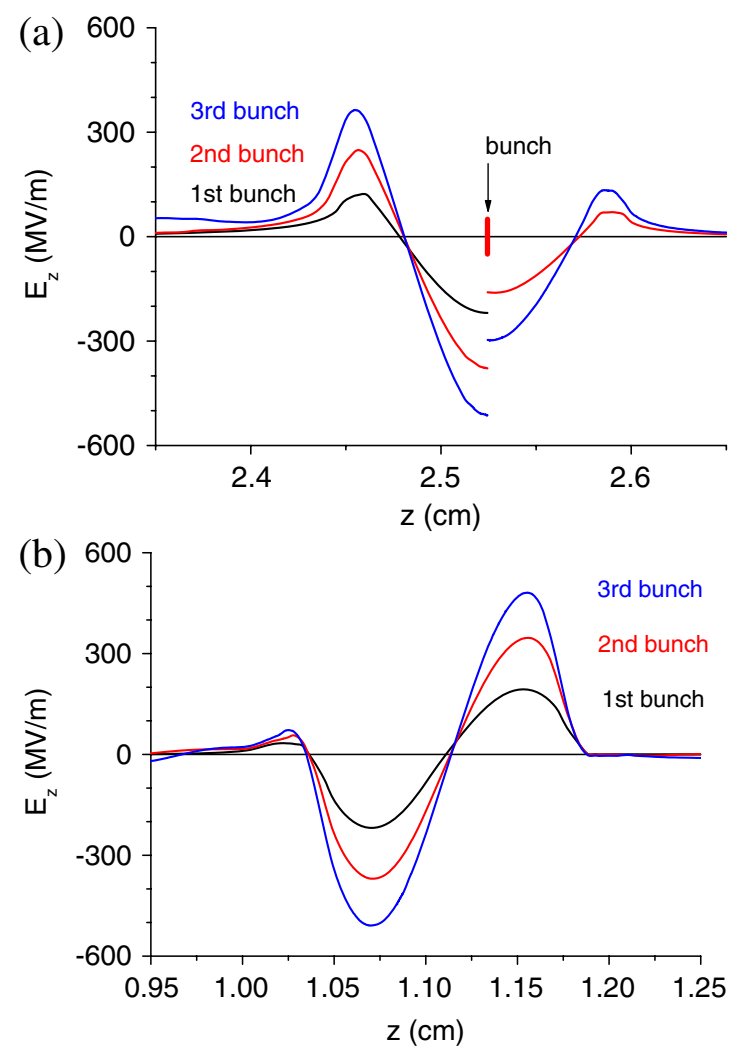

FIG. 14. (Color) The electric field on a beam line of the wakefield of multiple bunches: magnification of peaks of the curve in Fig. 13: (a) 1st peak (near bunch), (b) 2nd peak.

peak appears near the position of a beam bunch, and the second peak follows after $L=1.4 \mathrm{~cm}$. Figure 13(b) shows the acceleration gradient when the first bunch exits and the second bunch is located at the same position as the first bunch in Fig. 13(a). We chose the interval between the first bunch and the second bunch to satisfy the synchronization condition. In Fig. 13(b), one can notice that the magnitude

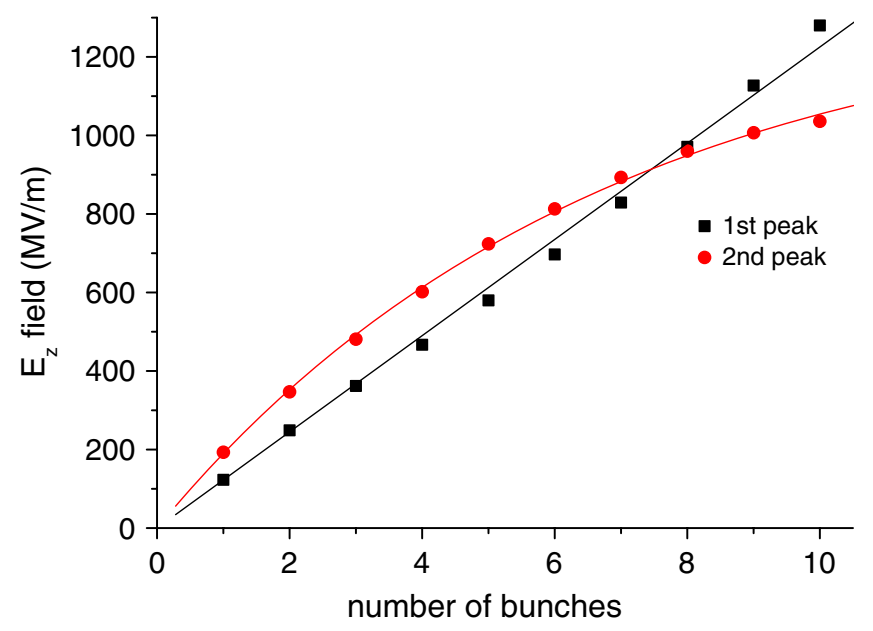

FIG. 15. (Color) Enhancement of the acceleration gradient with multiple bunches. of the acceleration gradient is increased. Also, the magnitude of the acceleration gradient by the third bunch is increased because it also satisfies the synchronization condition. Therefore, one can conclude that the wakefields in the dielectric-filled cavity are enhanced when the cavity and multibunches satisfy resonance and synchronization conditions.

Figure 14 is a magnified field plot of Fig. 13 near the two peaks. As shown in this figure, one can notice that field patterns of wakefields generated by multibunches maintain and their amplitudes get enhanced.

Figure 15 is a plot of positive peak values about the acceleration gradient for a number of bunches. For the first peak, its value linearly increases and the peak value of the acceleration gradient for the 10th bunch is $\sim 1.3 \mathrm{GV} / \mathrm{m}$. Also, the peak value of the acceleration gradient for the second peak becomes over $\sim 1.0 \mathrm{GV} / \mathrm{m}$.

\section{SUMMARY}

An analytical solution to the wakefield from a charge in a dielectric-filled cavity is derived. We introduced an image method for moving charge and used it to solve a cavity problem. The cavity wakefield was obtained by properly mirror reflecting the superimposed wakefields from the original beam created at the entrance time and the antibeam created at the exit time. The flux lines show that (1) the Cerenkov radiation is dominant in the dielectricfilled cavity and (2) the length of the Cerenkov radiation is independent of time. With these observations, we derived a resonance condition for dimensions of a cavity and a bunch-to-bunch period that efficiently enhances the wakefield for multiple bunches.

\section{ACKNOWLEDGMENTS}

The authors thank S.-d. Park for useful discussions. This work was supported by the BK21 program of the Korea Ministry of Education, Science and Technology (MEST).

[1] S. Y. Park and J.L. Hirshfield, Phys. Rev. E 62, 1266 (2000).

[2] M. Rosing and W. Gai, Phys. Rev. D 42, 1829 (1990).

[3] K.-Y. Ng, Phys. Rev. D 42, 1819 (1990).

[4] C. T. M. Chang and J. W. Dawson, J. Appl. Phys. 41, 4493 (1970).

[5] C. Wang and J. L. Hirshfield, Phys. Rev. ST Accel. Beams 9, 031301 (2006).

[6] I. N. Onishchenko, D. Y. Sidorenko, and G. V. Sotnikov, Phys. Rev. E 65, 066501 (2002).

[7] V. Balakirev, I. Onishchenko, D. Sidorenko, and G. Sotnikov, J. Exp. Theor. Phys. 93, 33 (2001).

[8] V. Balakirev, I. Onishchenko, D. Sidorenko, and G. Sotnikov, Tech. Phys. Lett. 29, 589 (2003).

[9] N. I. Onishchenko and G. Sotnikov, Tech. Phys. 53, 1344 (2008). 
[10] C. Wang, V.P. Yakovlev, T. C. Marshall, M. A. LaPointe, and J. L. Hirshfield, AIP Conf. Proc. 877, 910 (2006).

[11] A. Sommerfeld, Ann. Phys. (Leipzig) 349, 177 (1914).

[12] L. Brillouin, Ann. Phys. (Leipzig) 349, 203 (1914).

[13] A. Rubinowicz, Acta Phys. Pol. 10, 79 (1950).

[14] A. W. Chao, Physics of Collective Beam Instabilities in High Energy Accelerators (John Wiley \& Sons, New York, 1993).
[15] E. L. Burshtein and G. V. Voskresenskij, Zh. Tekh. Fiz. 33, 34 (1963) [Sov. Phys. Tech. Phys. 8, 22 (1963)].

[16] G. N. Watson, A Treatise on The Theory of Bessel Functions (Cambridge University Press, London, 1944), 2nd ed., p. 522.

[17] S. Y. Park and J.L. Hirshfield, Phys. Plasmas 8, 2461 (2001). 